\title{
What Future Studies are Needed for Obstructive Sleep Apnea Patients Undergoing Surgery?
}

\author{
Peter C. Gay*
}

Mayo Clinic College of Medicine, Rochester, MN, USA

This journal issue brought together many authors with a common interest in the care of patients undergoing surgical procedures requiring anesthesia who also have obstructive sleep apnea (OSA). This summary commentary refers to findings and discussions that took place at the October 15, 2010, Challenges in the Perioperative Management of OSA Patients conference. Anesthesiologists have been aware for decades of potential anesthetic-related complications that might be exaggerated in patients with OSA and the problem could be initially discovered in the post-anesthetic care unit (PACU) [1]. Most of these early studies were anecdotal in their scope and did not have enough impact to notably influence any change in patient care behavior. More controlled studies of larger post-operative patient populations suggested that the problem of OSA either 'unrecognized or untreated' could result in prolonged hospital stay and other respiratory complications [2].

The realization that patients with OSA could suffer undue post-operative consequences has led several investigator groups to begin to focus on ways to identify patients at risk for OSA. When the STOP portion of the STOP-BANG questionnaire was used in nearly 2,500 pre-surgical patients, it revealed that $28 \%$ were classified as being high risk for OSA, and follow-up PSG studies in a representative group of about 250 patients confirmed a high sensitivity for predicting OSA with AHI > 15 events/hour [3]. The predicted incidence of patients at high risk for OSA was much lower, near $4 \%$, when another questionnaire was used in a population of ambulatory surgery patients even though nearly 5\% were known to have previously diagnosed OSA [4]. Other authors have also showed that many different questionnaires can be utilized to predict which patients are at risk for OSA and also those likely to have post-operative complications with a high odds ratio $[5,6]$. This begs the question of what clinical tool or tools would best serve to identify patients at risk for OSA but more importantly, which patients might be more likely to have complications? This is a definite area in need of future studies to clarify remaining questions.

The National Center for Health Statistics Report from CDC on Outpatient Procedures in 2006 noted that outpatient procedures now account for about $2 / 3$ of all surgery visits in the U.S. [7]. Data from ambulatory patients indicates that visits increased from 20.8 million in 1996 to 34.7 million in 2006. During outpatient surgery, most frequently patients

\footnotetext{
*Address correspondence to this author at the Mayo Clinic College of Medicine, Rochester, MN, USA; Tel: 507-266-6880; Fax: 507-266-7772; E-mail: pgay@mayo.edu
}

underwent endoscopy (9.3 million) or cataract/lens surgery (5.7 million) and many of these patients were dismissed directly to home, often with narcotic medications. It will be important for future patient safety protocols to develop ways to manage patients that avoids potentially adverse consequences after dismissal from the hospital environment.

If we can predict who might have OSA and this leads to more post-operative complications, what should be done next? This easily provokes considerations about what is the optimal monitoring that should take place for patients at risk for OSA. The risk of hypoxemia and the need for pulse oximetry monitoring in both the operating room and the postanesthesia care unit are well recognized and have been formalized in treatment standards adopted by the ASA. One alternative is universal monitoring throughout the hospital in all post-operative patients but what is the evidence that supports universal monitoring for hypoxemia? Randomized trials have shown that oximetry can detect hypoxemia and other related events but there is little evidence to show that oximetry can reliably improve outcomes [8].

Perhaps oximetry is not the ideal monitoring technique and we need evidence to support the use of other monitoring approaches such as capnography. Must we have irrefutable evidence from randomized trials to justify any optimal monitoring technique? The use of $\mathrm{CO} 2$ monitoring may be an excellent point of discussion to illustrate why different levels of evidence might be required before a new technology should establish itself in practice. Capnography to confirm endotracheal tube placement has a simple and widely understood physiologic rationale. Presumably, only low level evidence is sufficient before one might recommend the widespread use of such a device if it is demonstrated that it is safe, cheap, sensitive, and specific [9]. On the other hand, end-tidal or sublingual capnometry are examples of $\mathrm{CO} 2$ monitoring that require very different levels of evidence before being widely disseminated. Gastric tonometry represents another situation where despite research supporting the use of this technology, the available data is not adequate to force a regularly established role in clinical practice.

Many questions remain regarding how post-surgical patients might best be monitored for averting potential complications. It could easily be argued that further scrutiny of optimal monitoring techniques will have little additional value without having a response to whatever monitoring pathway is chosen. This comment might best be supported by a reminder of what we learned from the past of possibly one of the most overused monitoring devices, that is pulmonary artery (PA) catheters. A report chronicled the marked decline 
in the use of PA catheters from 5.7 per 1000 medical admissions in 1993 to 2 per 1000 medical admissions in 2004 [10]. Clinicians began to reconsider their practice as it became apparent that complications seemed to outweigh benefits and there were no convincing randomized outcome trials to demonstrate a beneficial interventional behavior was associated with the PA catheter use. What was even more embarrassing was the fact that many clinicians were not as well versed as they perhaps should be to justify the frequency of use. A study was done using a multiple-choice exam administered to nearly 500 physicians practicing in the USA and Canada to assess their knowledge and understanding of PA catheter use and data interpretation [11]. The mean test score was $67 \%$ with a range of $19 \%$ to $100 \%$. The authors concluded that given the variability in physician understanding of the PA catheter, this warrants a credentialing policy reevaluation and consideration of restricted use. The defect here was likely not the misunderstanding of PA catheter use, but rather the lack of data guiding an appropriate response. Now, reconsider the present time where virtually every patient with suspected sepsis has a central venous (CV) catheter placed. Central venous catheters do have a purpose when they provide more than a number, but are tied to an intervention or specific pathway. In support of this was a large, clinical trial of early goal-directed therapy for the management of severe sepsis which is still the standard of care [12]. Once a clinical response is attached to a strong monitoring platform, positive outcome results will happen.

We need to be developing investigations to answer such crucial questions. We can see that the studies noted above, regarding the risk of post-operative disasters to OSA patients, support a surprisingly low number of serious complications, but there should be a different pursuit sought here. The challenge to us all should be refocused in another way. We have all seen that in any large institution that does a sufficiently frequent number of surgical procedures in patients with a high likelihood or known OSA, there will be serious postoperative complications and, no doubt in some cases, death may occur. Our goal should be a steadfast adherence to prevention because it only takes one unnecessary death or permanent anoxic encephalopathic event to urge a redesign of post-surgical practice [13]. Those insightful programs that offer comprehensive solutions to the potential problems in peri-operative patients with OSA will be the winners and a focus on management protocols of sleep disordered breathing in the surgical patient will be a strong starting point for improved healthcare delivery.

There remains an obvious need to support further clinical discussions in this area but also to better understand the basic differences and similarities between the states of consciousness while awake and during both sleep and while under the influence of anesthesia [14]. Perhaps the most fitting consequence of last year's Challenges in the Perioperative Management of OSA Patients conference gathering was that it served as the catalyst for the newly chartered Society of An- esthesia and Sleep Medicine (SASM). It is hoped that SASM will provide a forum for continued interactions regarding many common interests. Although this was supported by physicians from many different disciplines, this newly formed society was primarily the brainchild of Dr. Frances Chung from Toronto, Canada and Dr. David Hillman from Perth Australia. A steering committee was formed to construct bylaws and many have worked tirelessly to support the newly sculpted mission statement:

"The mission of SASM is to advance standards of care for clinical problems shared by Anesthesiology and Sleep Medicine, including peri-operative management of sleep disordered breathing, and to promote interdisciplinary communication, education and research in matters common to anesthesia and sleep."

\section{REFERENCES}

[1] Keamy MF III, Cadieux RJ, Kofke WA, Kales A. The occurrence of obstructive sleep apnea in a recovery room patient. Anesthesiology 1987; 66: 232-4.

[2] Gupta RM, Parvizij MD, Hanssen AD, Gay PC. Postoperative complications in patients with obstructive sleep apnea syndrome undergoing hip or knee replacement: a case-control study. Mayo Clin Proc 2001; 76: 897-905.

[3] Chung F, Yegneswaran B, Liao P, et al. STOP questionnaire: A tool to screen patients for obstructive sleep apnea. Anesthesiology 2008; 108: 812-21.

[4] Stierer TL, Wright C, George A, Thompson RE, Wu CL, Collop N. Risk assessment of obstructive sleep apnea in a population of patients undergoing ambulatory surgery. J Clin Sleep Med 2010; 6: 467-72.

[5] Chung F, Yegneswaran B, Liao P, et al. Validation of the Berlin Questionnaire and American Society of Anesthesiologists Checklist as screening tools for obstructive sleep apnea in surgical patients. Anesthesiology 2008; 108: 822-30.

[6] Gali B, Whalen FX, Schroeder DR, Gay PC, Plevak DJ. Identification of patients at risk for postoperative respiratory complications using a preoperative obstructive sleep apnea screening tool and postanesthesia care assessment. Anesthesiology 2009; 110(4): 86977.

[7] http://www.google.com/search?q=National+Center+for + Health+ Statistics + Report + from $+\mathrm{CDC}+$ on + Outpatient + Procedures\&rls $=$ com.microsoft:en-us\&ie $=U \mathrm{UTF}-8 \&$ oe $=\mathrm{UTF}-8 \&$ startIndex $=\&$ start Page $=1$ [Accessed 12/12/10].

[8] Pedersen T. Does perioperatuic pulse oximetry improve outcome? seeking the best available evidence to answer the clinical question. Best Pract \& Res Clin Anesthesiol 2005; 19(1): 111-23.

[9] Povoas HP, Weil MH, Tang W, et al. Comparisons between sublingual and gastric tonometry during hemorrhagic shock. Chest 2000, 118: 1127-32.

[10] Wiener RS, Welch HG. Trends in the use of the pulmonary artery catheter in the United States, 1993-2004. JAMA 2007; 298(4): 423-9.

[11] Iberti T, Fischer E, Leibowitz M, et al. A multicenter study of physicians' knowledge of the pulmonary artery catheter. JAMA 1990; 264: 2928-32.

[12] Rivers E, Nguyen B, Havstad S, et al. Early goal-directed therapy in the treatment of severe sepsis and septic shock. N Engl J Med 2001; 345: 1368-77.

[13] Gay PC. The value of assessing risk of obstructive sleep apnea in surgical patients: it only takes one. J Clin Sleep Med 2010; 6: 467-72.

[14] Chamberlin NL, Eikermann M. This is no humbug: anesthetic agent-induced unconsciousness and sleep are visibly different. Anesthesiology 2010;113(5): 1007-9. 